\title{
Hormones of Bone Metabolism Changed with Vitamin D Agents Treatment in Chronic Kidney Disease Patients
}

\author{
Dongliang Zhang ${ }^{1 *}$, Xiaodan Sun ${ }^{2}$ and $\mathrm{He} \mathrm{Li}^{3}$
}

\author{
${ }^{1}$ Blood Purification Center of Nephrology Department, Peking University International Hospital, P.R. China
}

${ }^{2}$ Department of Nephrology, Beijing Rehabilitation Hospital of Capital Medical University, P.R. China

${ }^{3}$ Department of Nephrology, Civil Aviation General Hospital, P. R. China

\begin{abstract}
Objective: To compare the circulation levels of hormones related with bone metabolism in a cohort of patients with chronic kidney disease in stage 3-5 who were treated by ergocalciferol (VitD2) or calcitriol (aVitD3).

Methods: A post-hoc study was designed, and 204 patients were enrolled into the present study with a mean following-up duration of $33.2 \pm 3.8$ months. Patients in Group VitD2 $(n=104)$ and Group aVitD3 $(n=100)$ were treated by ergocalciferol and calcitriol, respectively. The circulation levels of routine biochemical parameters were detected including hemoglobin $(\mathrm{Hb})$, hypersensitive $\mathrm{C}$ reactive protein (hs-CRP), serum creatinine ( $\mathrm{SCr}$ ), albumin $(\mathrm{A})$, total cholesterol (TCh), triglycerides (TG), calcium (Ca), phosphorus (P), as well as bone biomarkers covering alkaline phosphatase (ALP), intact parathyroid hormone (iPTH), 25-hydroxyvitamin D, fibroblast growth factor-23 (FGF-23), klotho, bone morphogenetic protein (BMP) and osteopontin (OPN). Statistic analysis was conducted on parameters levels changes according to different time points and groups during following up.

Results: Among different time points, there were significant differences in the circulating levels of $\mathrm{Ca}(F=2.908, P=0.014)$, $\mathrm{P}(F=9.168, P<0.001), \mathrm{ALP}(F=4.625, P=0.005)$, iPTH $(F=4.064, P=0.003), 25(\mathrm{OH}) \mathrm{D}(F=306.48, P<0.001), \mathrm{FGF}-23$ $(F=118.42, P<0.001)$, klotho protein $(F=71.079, P<0.001)$, and OPN $(F=6.576, P<0.001)$. The $25(\mathrm{OH}) \mathrm{D}(F=209.222$, $P<0.001)$ and BMP-7 $(F=44.245, P<0.001)$ levels of VitD2 group increased significantly than aVitD3 group during treatment. 25(OH) D level had a significantly negative correlation with FGF-23 after 24 months vitamin D supplements treatment $(t=-2.572, \beta=-0.177, P=0.011)$.
\end{abstract}

Conclusions: The blood levels of bone metabolism hormones change significantly after vitamin D agents' therapy. Ergocalciferol supplementation treatment can increase blood levels of 25(OH) D and BMP-7 much more than calcitriol. Improving 25(OH) D insufficiency can suppress the endocrine of FGF-23.

Keywords

Bone morphogenetic protein-7, Calcitriol, Chronic kidney disease, Ergocalciferol, Osteopontin

\section{Introduction}

Chronic kidney disease mineral bone disorder (CKD$\mathrm{MBD}$ ) is highly prevalent in CKD stage 3-5 patients $[1,2]$. Though bone biopsy can give an identify diagnosis of CKD-MBD, it is difficult for mostly hospitals to conduct the biopsy due to patients' reluctance. Moreover, at an early stage of CKD-MBD, the only signs of the disease may be abnormal levels and bioactivities of the bone metabolism hormones and circulation biochemical markers, which including blood calcium $(\mathrm{Ca})$, phosphorus $(\mathrm{P})$, parathyroid hormone $(\mathrm{PTH})$, alkaline phosphatase (ALP), Vitamin D [3,4], fibroblast growth factor 23 (FGF-23) and klotho, etc. [5]. Thus, the circulation bio- markers of CKD-MBD are very important. Besides blood biomarkers on bone metabolism including bone special alkaline phosphatase (BALP), osteopontin (OPN),

\begin{abstract}
*Corresponding author: Dongliang Zhang, Blood Purification Center of Nephrology Department, Peking University International Hospital, No.1 Life Park Road, Zhongguancun Life Park, Changping District, Beijing, China, Tel: +86-1069006549
\end{abstract}

Accepted: November 14, 2018:

Published online: November 16, 2018

Citation: Zhang D, Sun X, Li H (2018) Hormones of Bone Metabolism Changed with Vitamin D Agents Treatment in Chronic Kidney Disease Patients. J Hum Nutr 2(1):47-57

Copyright: (C) 2018 Zhang D, et al. This is an open-access article distributed under the terms of the Creative Commons Attribution License, which permits unrestricted use, distribution, and reproduction in any medium, provided the original author and source are credited. 
Citation: Zhang D, Sun X, Li H (2018) Hormones of Bone Metabolism Changed with Vitamin D Agents Treatment in Chronic Kidney Disease Patients. J Hum Nutr 2(1):47-57

bone morphogenetic protein (BMP), and FGF-23 could also provide very important information and evidence for clinical assessment of CKD-MBD. The correlations among these bone metabolism hormones are complex, which need further research. For example, overloaded phosphate deposition causes arterial medial calcification, while OPN and FGF-23 may be inducers and markers of this process [6-9]. Increased extracellular phosphate and vitamin D hormone stimulate the secretion of BMP7 and FGF-23 in bone, which forming a feedback loop between bone and kidney [10].

In CKD patients, vitamin D insufficiency and deficiency are more common than in the general population [11]. The prevalence of 25 -hydroxyvitamin D [25(OH) D] deficiency in adults at CKD stage 2-4 has been reported at $71 \%-82 \%$ and is closely correlated with CKD stages [12]. Vitamin D deficiency plays a critical role in CKD-MBD and is associated with mortality and mobility in CKD patients [3,4]. Therefore, vitamin D supplements are common in the treatment of CKD-MBD. To compare the efficacy and safety of ergocalciferol (vitamin D2, Vit D2) and calcitriol (activated vitamin D3, aVitD3) on treatment of secondary hyperparathyroidism (sHPT) in stage 3-5 CKD patients, we conducted a randomized, prospective, controlled, open-labeled study [13]. Because it remains unclear whether the bone metabolism biomarkers respond to vitamin $\mathrm{D}$ therapies, we also detected the changes of circulating levels for bone metabolism biomarkers, including ALP, FGF-23, klotho protein, BMP-7, and OPN for two years following-up. The present post-hoc study would provide valuable information for controlling CKD-MBD.

\section{Methods}

\section{Study design}

The protocol was detailed in our previous work [13]. A total of 254 patients were recruited from January 2011 to June 2012, and 204 patients finished the following-up until June 2014 (Figure 1). Subjects were randomized into two groups: oral ergocalciferol (Group VitD2) treatment group and calcitriol (Group aVitD3) treatment group. These enrolled patients were visited every 3 months in the clinic during follow-up period, and underwent routine measurements of hemoglobin $(\mathrm{Hb})$, hypersensitive $\mathrm{C}$ reactive protein (hs-CRP), serum creatinine ( $\mathrm{SCr}$ ), albumin (A), total cholesterol (TCh), triglycerides (TG), $\mathrm{Ca}, \mathrm{P}, \mathrm{ALP}$, and iPTH. Estimated glomerular filtration rate (eGFR) was calculated using the Modification of Diet

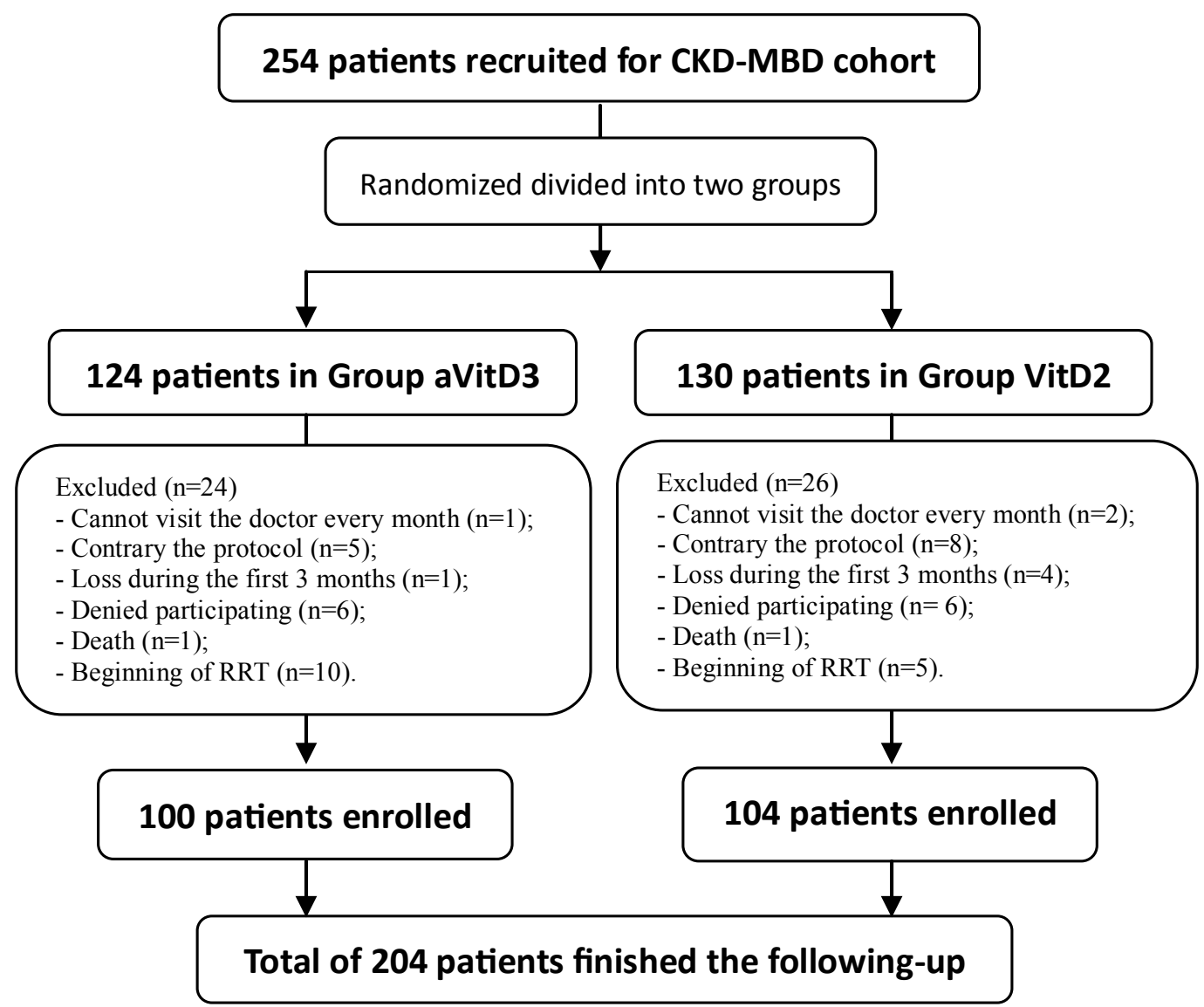

Figure 1: Flow chart of participants in the cohort. CKD-MBD, chronic kidney disease mineral bone disorders; aVitD3, active vitamin D3, patients in this group were treated by calitriol; VitD2, vitamin D2, patients in this group were treated by ergocalciferol; RRT, renal replacement therapy. 
Citation: Zhang D, Sun X, Li H (2018) Hormones of Bone Metabolism Changed with Vitamin D Agents Treatment in Chronic Kidney Disease Patients. J Hum Nutr 2(1):47-57

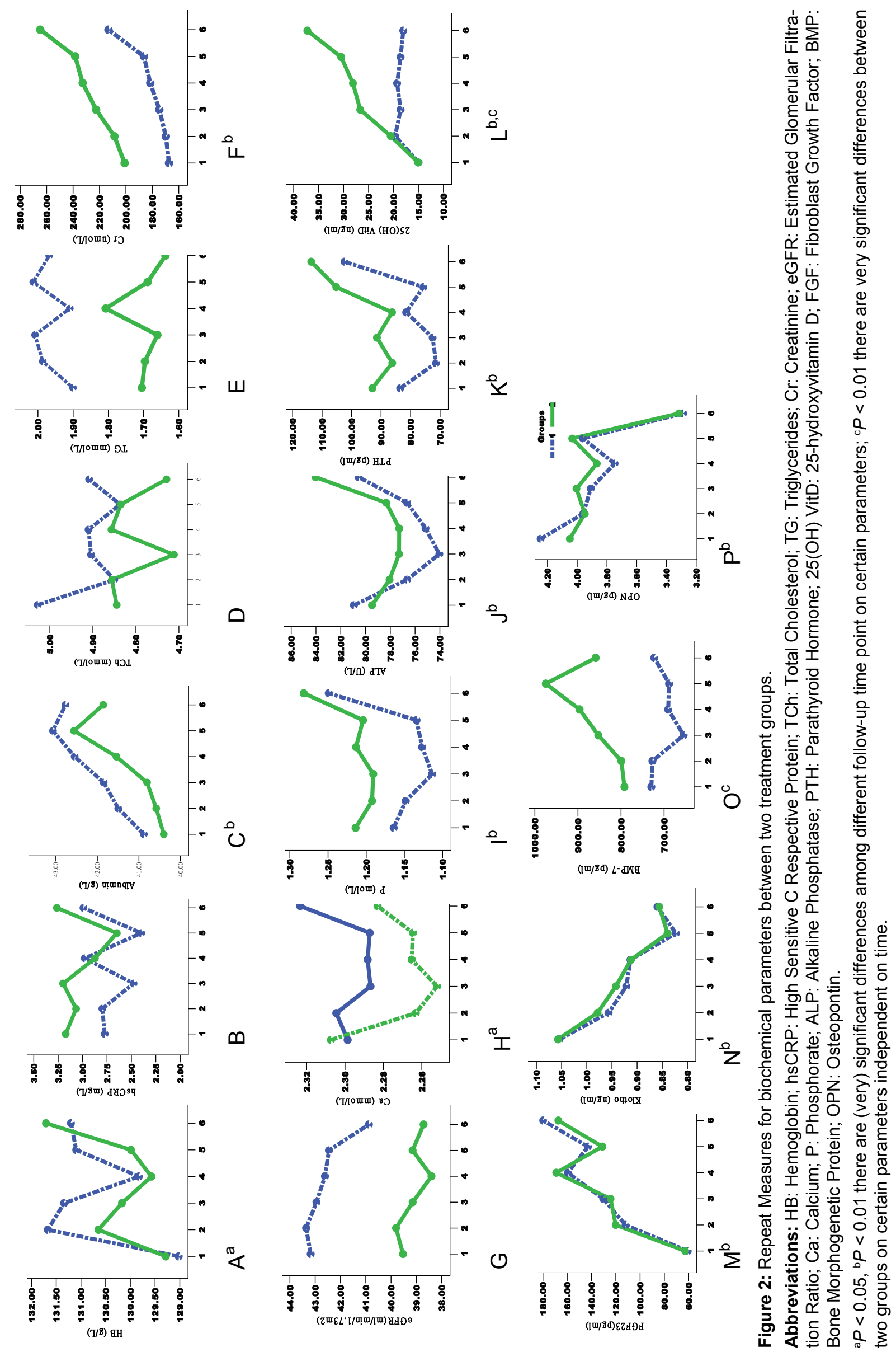


Citation: Zhang D, Sun X, Li H (2018) Hormones of Bone Metabolism Changed with Vitamin D Agents Treatment in Chronic Kidney Disease Patients. J Hum Nutr 2(1):47-57

in Renal Disease (MDRD) formula. Blood levels of bone metabolism hormones were measured every 6 months, including FGF-23, klotho protein, BMP-7, OPN, as well as $25(\mathrm{OH}) \mathrm{D}$.

The target level of $25(\mathrm{OH}) \mathrm{D}$ was $20 \mathrm{ng} / \mathrm{ml}$ in this study. The clinical outcomes included maintenance target levels of serum $\mathrm{Ca}, \mathrm{P}, \mathrm{iPTH}$ and $25(\mathrm{OH}) \mathrm{D}$ according to the Kidney Disease Outcomes Quality Initiative (KDOQI) clinical practice guidelines [3]. The primary end-point events included all-cause death (ACD), acute non-fatal myocardial infarction (AMI), acute non-fatal stroke, congestive heart failure (CHF), and non-traumatic fracture (NTF). The secondary end-point events were the beginning of renal replacement therapy (RRT), eGFR decline $>25 \%$, or doubling in SCr levels.

\section{Biochemical assays}

Whole blood samples were obtained regularly, and the serum samples were collected, and frozen immediately at $-80^{\circ} \mathrm{C}$ until they were analyzed. The serum levels of $25(\mathrm{OH}) \mathrm{D}$ were measured by using high-performance liquid chromatography-tandem mass spectrometry (LCMS/MS) [14] (Sciex API 2000 MDS LC-MS System, Applied Biosystems, USA). Serum FGF-23, klotho protein, BMP-7 and OPN were measured using enzyme linked immunosorbent assay (ELISA) kits (Blue Gene Biotech, China). The routine investigation parameters were measured by standard methods.

\section{Statistical analysis}

The patients' characteristics and parameters are summarized by treatment groups. The results are presented as mean \pm SD for continuous variables and frequency for categorical variables. In order to identify the differences of biomarkers' mean levels during follow-up whether caused by different treatments or by different time points, we used General Linear Model Repeated Measures for continuous variables. To observe the complex relationships among the bone metabolism biomarkers before (Month 0) and after (Month 24) vitamin D supplements treatment, it was calculated by Pearson's test and thereafter Linear Regression analyses. The predict factors among these biomarkers for either the outcomes or endpoint events were detected by Logistic Regression, respectively. $P<0.05$ was considered to indicate a significant difference. All of the analyses were performed using SPSS software (version 13.0 for Windows; SPSS Inc., Chicago, IL, USA).

The study protocol was approved by central ethical review boards of the principal investigator's clinical facility. The study was conducted in accordance with the principles of the Declaration of Helsinki (ClinicalTrials. gov identifier: NCT01633853).

\section{Results}

In these 204 individuals who finished the following up, 104 were assigned to the VitD2 group, and 100 were assigned to the aVitD3 group. The mean duration of follow-up was $33.2 \pm 3.8$ months (23.3-39.9 months). The baseline patient characteristics were shown in our previous published article [13]. Table 1 show the biochemical parameters levels in different treatment groups.

All 204 patients completed 24 months follow-up, and there were 177 patients $(86.8 \%)$ finished 30 months follow-up. The repeated measures of General Linear Model were calculated according to the data for 30 months follow-up. In regular laboratory parameters, the levels of $\mathrm{Hb}, \mathrm{A}$, and SCr changed significantly with elevated trends during following up (Table 1, Figure 2A, Figure $2 \mathrm{C}$ and Figure $2 \mathrm{~F}$ ). Other parameters, such as hsCPR, TCh, TG, and eGFR, did not show significant changes among different time points. For bone metabolism biomarkers, there were significant differences in the circulating levels of $\mathrm{Ca}, \mathrm{P}, \mathrm{ALP}, \mathrm{iPTH}, 25(\mathrm{OH}) \mathrm{D}, \mathrm{FGF}-23$, klotho protein, as well as OPN over time. (Table 1, Figure 2H, Figure 2I, Figure 2J, Figure 2K. Figure 2L, Figure $2 \mathrm{M}$, Figure $2 \mathrm{~N}$ and Figure $2 \mathrm{P}$ ). In details, the BMP7 levels changed without significant difference (Table 1 and Figure 2O). The trends of Ca, P, ALP, and iPTH levels showed " $U$ " shape, which the values decreased at the $0,6^{\text {th }}$, and $12^{\text {th }}$ month, then increased gradually in later follow-up period (Figure 2). Both 25(OH) D and FGF-23 levels increased significantly during following up, in contrast that both Klotho protein and OPN levels decreased significantly. When compared the $25(\mathrm{OH}) \mathrm{D}$ and BMP-7 levels between two groups, their levels of the VitD2 group increased significantly than aVitD3 group (Table 1, Figure 2L and Figure 2O). All other biochemical parameters of bone metabolism showed no significant differences between the two groups (Table 1 and Figure 2).

According to the Linear Regression tests, there were complex correlations among these parameters. On baseline, $\mathrm{Ca}$ had significant correlation with $\mathrm{A}, \mathrm{TCh}$, and iPTH, while $\mathrm{P}$ was correlated with $\mathrm{Hb}, \mathrm{SCr}$, eGFR and OPN, CRP was correlated with ALP, iPTH was correlated with Ca and SCr, BMP-7 was correlated with klotho protein, OPN was correlated with $\mathrm{P}$ and BMP-7, as well as BMP-7 was correlated with $\mathrm{Hb}$, klotho protein and $\mathrm{OPN}$, respectively (Table 2 and Figure $3 \mathrm{~A}$ ). Neither 25(OH) D nor FGF-23 had statistically significant correlations among other parameters on baseline. On the $24^{\text {th }}$ follow-up month, the significant correlations existed as: $\mathrm{Ca}$ with $\mathrm{SCr}$ and $\mathrm{TCh}, \mathrm{P}$ with $\mathrm{Hb}$ and $\mathrm{SCr}$, ALP with CRP and $\mathrm{Ca}$, iPTH with $\mathrm{Ca}, \mathrm{SCr}$, and eGFR, 25(OH) D with FGF-23 and BMP-7, 25(OH) D with FGF-23, Ca with klotho protein, as well as $25(\mathrm{OH})$ D with BMP-7, 
Citation: Zhang D, Sun X, Li H (2018) Hormones of Bone Metabolism Changed with Vitamin D Agents Treatment in Chronic Kidney Disease Patients. J Hum Nutr 2(1):47-57

\begin{tabular}{|c|c|c|c|c|c|c|c|c|c|c|c|c|c|c|c|c|c|}
\hline 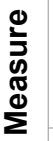 & 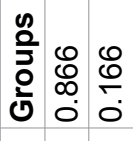 & $\frac{n}{\mathfrak{d}}$ & $\begin{array}{l}8 \\
0 \\
0\end{array}$ & $\begin{array}{l}L \\
0 \\
0 \\
0\end{array}$ & $\begin{array}{l}\stackrel{Q}{N} \\
\stackrel{m}{m}\end{array}$ & 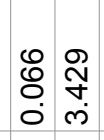 & 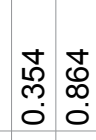 & $\begin{array}{l}8 \\
8 \\
0 \\
0\end{array}$ & . & $\begin{array}{l}\text { ¿ } \\
0\end{array}$ & 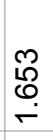 & & 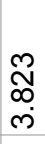 & Oे & $\begin{array}{l}\mathscr{O} \\
\infty \\
i\end{array}$ & & ণ \\
\hline व & 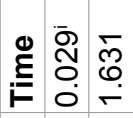 & $\begin{array}{l}0 \\
\text { L } \\
0 \\
0\end{array}$ & 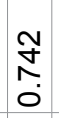 & $\begin{array}{l}\bar{\delta} \\
0 \\
0 \\
v\end{array}$ & $\begin{array}{l}9 \\
\stackrel{9}{f} \\
\infty \\
\infty \\
\leftarrow\end{array}$ & 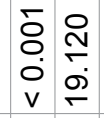 & $\begin{array}{ll}0 & 2 \\
0 & 8 \\
0 & 0 \\
0 & 0\end{array}$ & $\begin{array}{l}\mathscr{8} \\
8 \\
\infty \\
0\end{array}$ & $\frac{N}{\tilde{N}}$ & 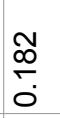 & 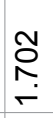 & & $\begin{array}{l}\infty \\
\stackrel{8}{S} \\
\text { in }\end{array}$ & & $\frac{\infty}{\varnothing}$ & & $\begin{array}{l}\text { ָे } \\
\stackrel{+}{+}\end{array}$ \\
\hline & $0<$ & 2 & 4 & a & $\psi$ & $0<$ & a L L & $a$ & 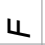 & $a$ & $L$ & $a$ & $L$ & 0 & $L$ & a & 4 \\
\hline
\end{tabular}
৪

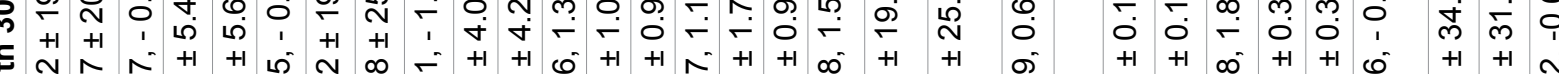

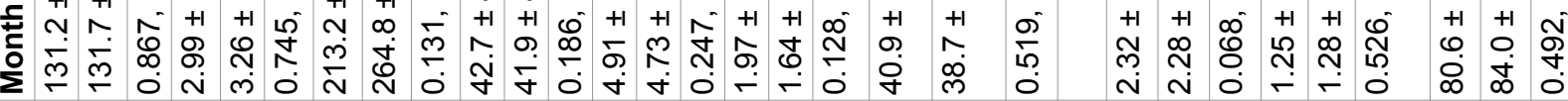

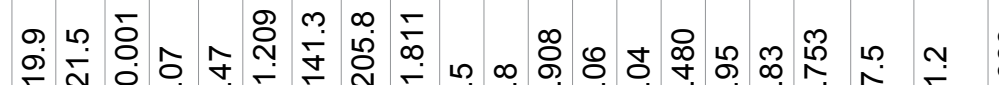

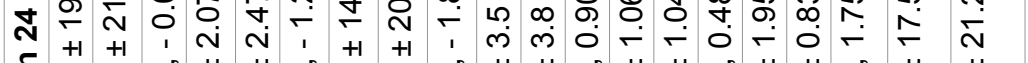
艺 ¿

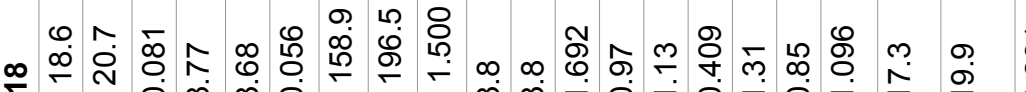

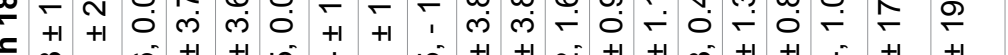

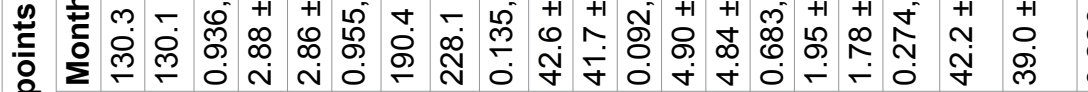
$\stackrel{\oplus}{\xi}$

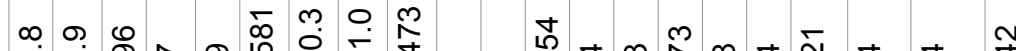

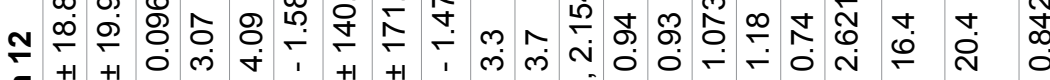

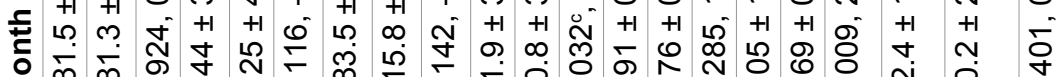

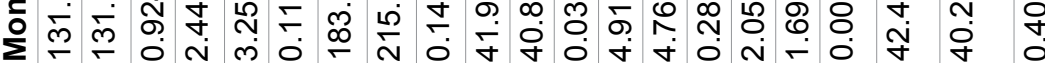

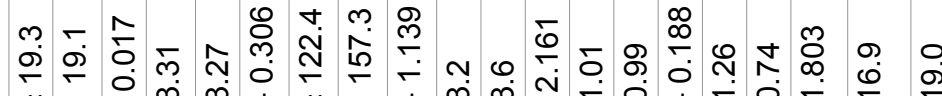

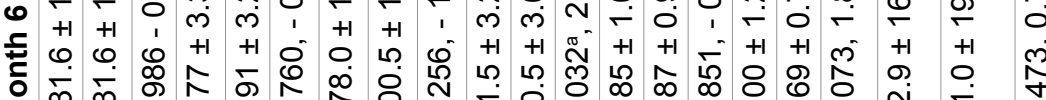

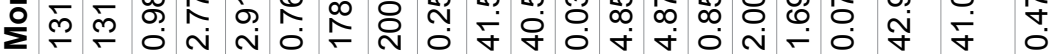

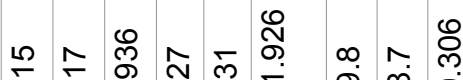

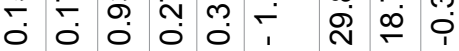

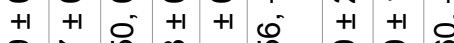
న సิ n
ஸு

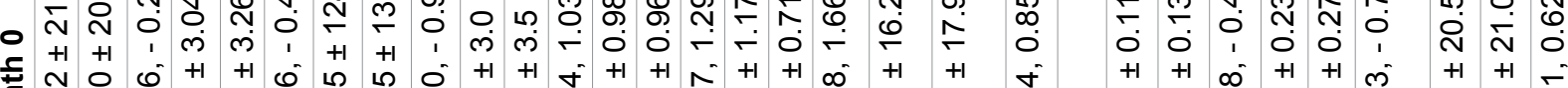

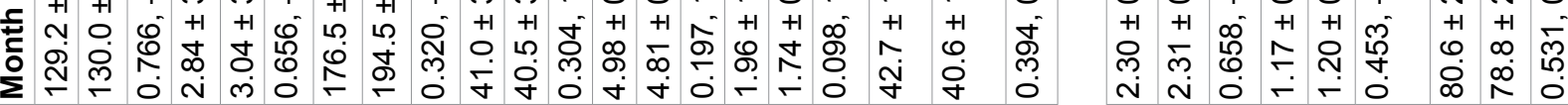

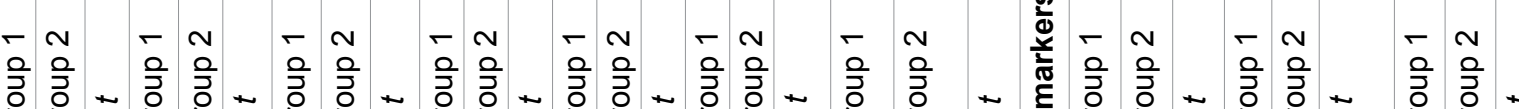
焉

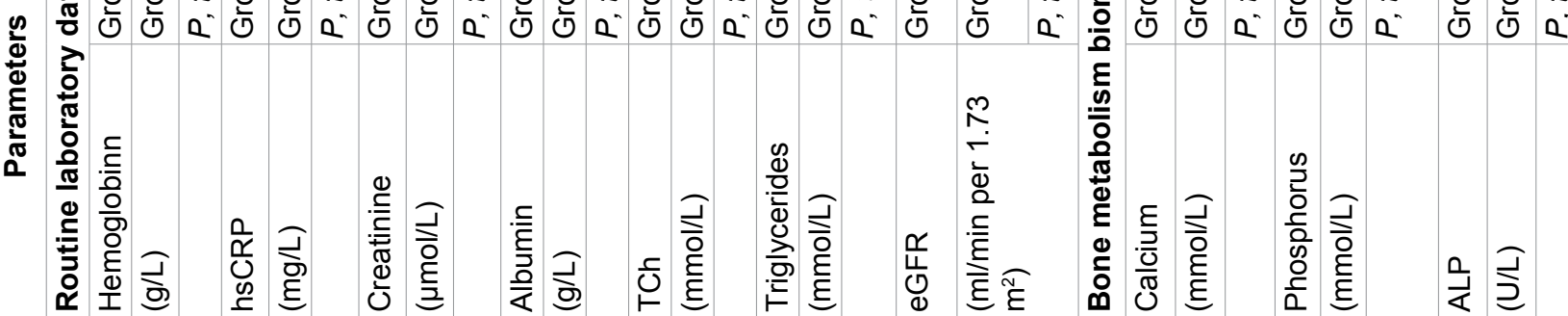




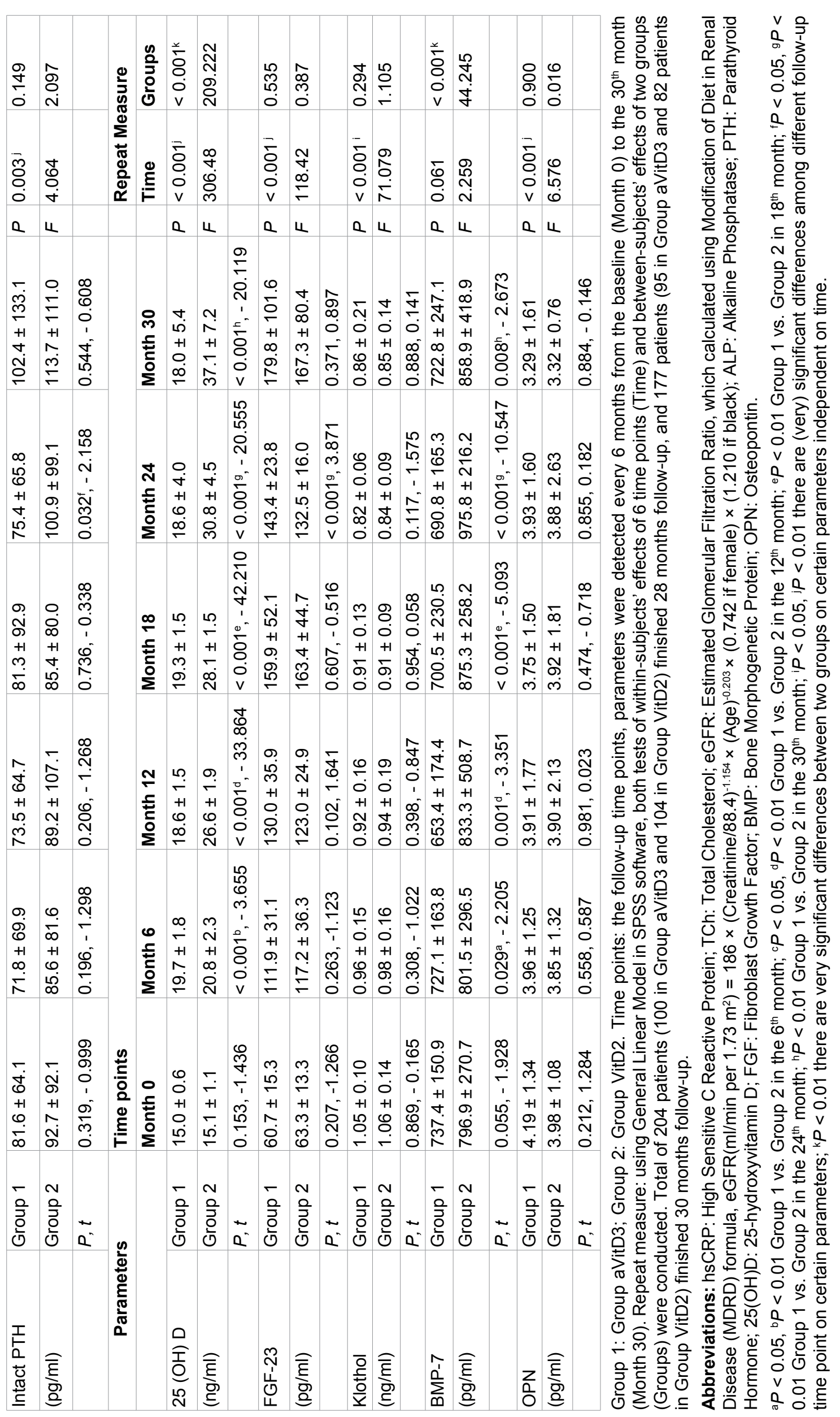


Citation: Zhang D, Sun X, Li H (2018) Hormones of Bone Metabolism Changed with Vitamin D Agents Treatment in Chronic Kidney Disease Patients. J Hum Nutr 2(1):47-57

respectively (Table 2 and Figure 3B). There were no significant correlations among OPN and other parameters.

Furthermore, there were no biomarkers of bone metabolism affecting any primary endpoint events. However, $\mathrm{P}(P<0.001, \mathrm{OR}=10.825)$ and $\mathrm{iPTH}(P=0.025, \mathrm{OR}=$ $1.005)$ levels of baseline could predict the secondary endpoint events (beginning of RRT, eGFR decline > 25\%, and doubling in SCr levels).

\section{Discussion}

Both KDOQI and KDIGO guidelines for CKD-MBD recommend using nutritional vitamin $\mathrm{D}$ supplements to treat CKD-MBD patients [3,4]. In one of our previous studies [13], the results suggested ergocalciferol has the similar effects with calcitriol on controlling CKD-MBD by maintaining the target levels of $\mathrm{Ca}, \mathrm{P}$, and $\mathrm{iPTH}$ in CKD stage 3-5 patients. We also found the circulation level changes of bone metabolism hormones, such as
FGF-23, klotho protein, OPN and BMP-7, in this randomized 36-month study. Though several previous experiments studied on the relationship of calcitriol and these hormones, such as FGF-23, klotho and BMP-7, as we know, this is the first time to report the effects of long-term treatment by different vitamin $\mathrm{D}$ agents on bone metabolism hormones. The results may provide some information for the treatment of CKD-MBD.

In CKD-MBD patients, one of the aims of vitamin $\mathrm{D}$ supplements is increase the level of vitamin D. The present study identified that ergocalciferol supplement could raise the blood level of $25(\mathrm{OH}) \mathrm{D}$ more significantly than calcitriol (Table 1 and Figure 2L). Most patients in Group VitD2 $(96 / 104,90.4 \%)$ could reach the target of 25(OH) D level during the first 6 months, and the level increased continuously over time. The blood level of $25(\mathrm{OH}) \mathrm{D}$ also increased during early period in Group aVitD3 but declined later. Possible explanation

Table 2: The correlations among bone metabolism biomarkers.

\begin{tabular}{|c|c|c|c|c|c|}
\hline \multicolumn{2}{|c|}{$\begin{array}{l}\text { Bone metabolism } \\
\text { biomarkers }\end{array}$} & \multicolumn{4}{|l|}{ Correlated parameters } \\
\hline $\mathrm{Ca}$ & Month 0 & A & TCh & iPTH & \\
\hline \multirow[t]{3}{*}{$(\mathrm{mmol} / \mathrm{L})$} & $P, t, \beta$ & $0.020^{a}, 2.345,0.156$ & $0.012^{a}, 2.536,0.170$ & $0.001^{\mathrm{b}},-3.409,-0.229$ & \\
\hline & Month 24 & $\mathrm{SCr}$ & TCh & & \\
\hline & $P, t, \beta$ & $<0.001^{a}, 3.909,0.315$ & $<0.001^{a},-4.098,0.328$ & & \\
\hline $\mathrm{P}$ & Month 0 & $\mathrm{Hb}$ & $\mathrm{SCr}$ & eGFR & OPN \\
\hline \multirow[t]{3}{*}{$(\mathrm{mmol} / \mathrm{L})$} & $P, t, \beta$ & $0.003^{b}, 2.345,0.156$ & $<0.001^{\mathrm{b}}, 3.962,0.347$ & $0.042^{\mathrm{a}},-2.048,-0.188$ & $0.018^{a}, 2.385,0.134$ \\
\hline & Month 24 & $\mathrm{Hb}$ & $\mathrm{SCr}$ & & \\
\hline & $P, t, \beta$ & $0.007^{b},-2.710,-0.168$ & $<0.001^{\mathrm{b}}, 6.009,0.492$ & & \\
\hline ALP & Month 0 & CRP & & & \\
\hline \multirow[t]{3}{*}{$(\mathrm{U} / \mathrm{L})$} & $P, t, \beta$ & $<0.001^{\mathrm{b}}, 5.007,0.332$ & & & \\
\hline & Month 24 & CRP & $\mathrm{Ca}$ & & \\
\hline & $P, t, \beta$ & $<0.001^{\mathrm{b}}, 5.599,0.365$ & $0.016^{a},-2.419,-0.155$ & & \\
\hline iPTH & Month 0 & $\mathrm{Ca}$ & $\mathrm{SCr}$ & & \\
\hline \multirow[t]{3}{*}{$(\mathrm{pg} / \mathrm{ml})$} & $P, t, \beta$ & $<0.001^{\mathrm{b}},-4.552,-0.281$ & $0.002^{b}, 3.140,0.311$ & & \\
\hline & Month 24 & $\mathrm{Ca}$ & $\mathrm{SCr}$ & eGFR & \\
\hline & $P, t, \beta$ & $<0.001^{\mathrm{b}},-4.794,-0.271$ & $<0.001^{\mathrm{b}}, 4.339,0.416$ & $0.024^{a},-2.277,-0.219$ & \\
\hline $25(\mathrm{OH}) \mathrm{D}$ & Month 0 & None & & & \\
\hline \multirow[t]{3}{*}{$(\mathrm{ng} / \mathrm{ml})$} & $P, t, \beta$ & & & & \\
\hline & Month 24 & FGF-23 & BMP-7 & & \\
\hline & $P, t, \beta$ & $0.024^{a},-2.272,-0.135$ & $<0.001^{\mathrm{b}}, 8.644,0.514$ & & \\
\hline FGF-23 & Month 0 & None & & & \\
\hline \multirow[t]{3}{*}{$(\mathrm{pg} / \mathrm{ml})$} & $P, t, \beta$ & & & & \\
\hline & Month 24 & $25(\mathrm{OH}) \mathrm{D}$ & & & \\
\hline & $P, t, \beta$ & $0.011^{a},-2.572,-0.177$ & & & \\
\hline Klotho & Month 0 & BMP-7 & & & \\
\hline \multirow[t]{3}{*}{ (ng/ml) } & $P, t, \beta$ & $<0.001^{\mathrm{b}}, 3.756,0.254$ & & & \\
\hline & Month 24 & $\mathrm{Ca}$ & & & \\
\hline & $P, t, \beta$ & $0.034^{a},-2.130,-0.148$ & & & \\
\hline OPN & Month 0 & $\mathrm{P}$ & BMP-7 & & \\
\hline \multirow[t]{3}{*}{$(\mathrm{pg} / \mathrm{ml})$} & $P, t, \beta$ & $0.006^{b}, 2.792,0.184$ & $<0.001^{b},-4.590,-0.302$ & & \\
\hline & Month 24 & \multirow[t]{2}{*}{ None } & & & \\
\hline & $P, t, \beta$ & & & & \\
\hline
\end{tabular}


Citation: Zhang D, Sun X, Li H (2018) Hormones of Bone Metabolism Changed with Vitamin D Agents Treatment in Chronic Kidney Disease Patients. J Hum Nutr 2(1):47-57

\begin{tabular}{|l|l|l|l|l|}
\hline BMP-7 & Month 0 & $\mathrm{Hb}$ & Klotho & OPN \\
\hline$(\mathrm{ng} / \mathrm{ml})$ & $P, t, \beta$ & $0.023^{\mathrm{a}}, 2.285,0.147$ & $<0.001^{\mathrm{b}}, 3.897,0.250$ & $<0.001^{\mathrm{b}},-4.575,-0.295$ \\
\hline & Month 24 & $25(\mathrm{OH}) \mathrm{D}$ & & \\
\hline & $P, t, \beta$ & $<0.001^{\mathrm{b}}, 8.827,0.528$ & & \\
\hline
\end{tabular}

The significant correlated parameters of each bone metabolism biomarkers in month 0 and 24 were listed.

Abbreviations: Hb: Hemoglobin; hsCRP: High Sensitive C Reactive Protein; A: Albumin; SCr: Serum Creatinine; eGFR: Estimated Glomerular Filtration Ratio; TCh: Total Cholesterol; TG: Triglycerides; Ca: Calcium; P: Phosphorus; ALP: Alkaline Phosphatase; iPTH: Intact Parathyroid Hormone; 25(OH)D: 25-hydroxyvitamin D; FGF: Fibroblast Growth Factor; BMP: Bone Morphogenetic Protein; OPN: Osteopontin; $\beta$ : Standardized Coefficient. ${ }^{\text {a }} P<0.05,{ }^{\text {b }} P<0.01$.

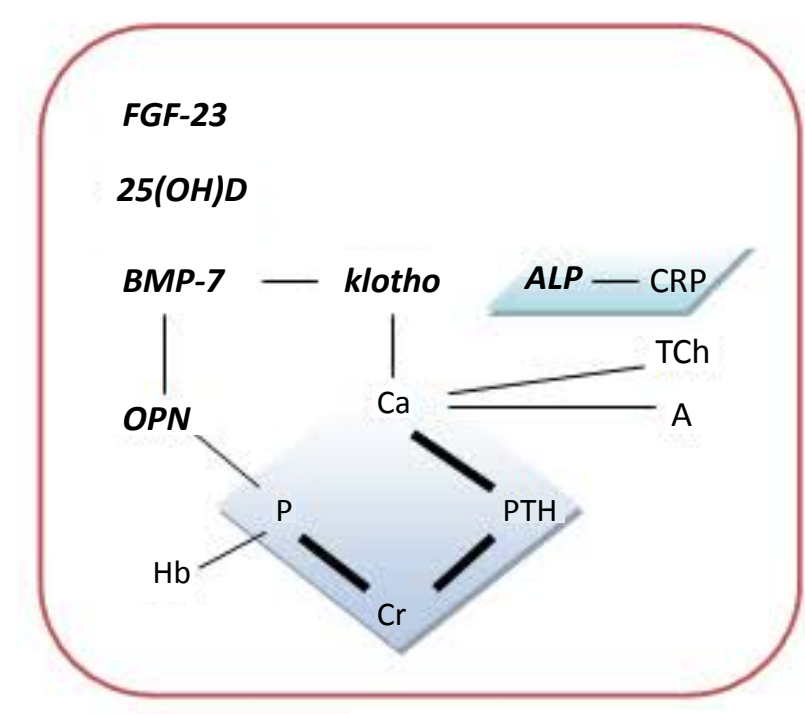

A

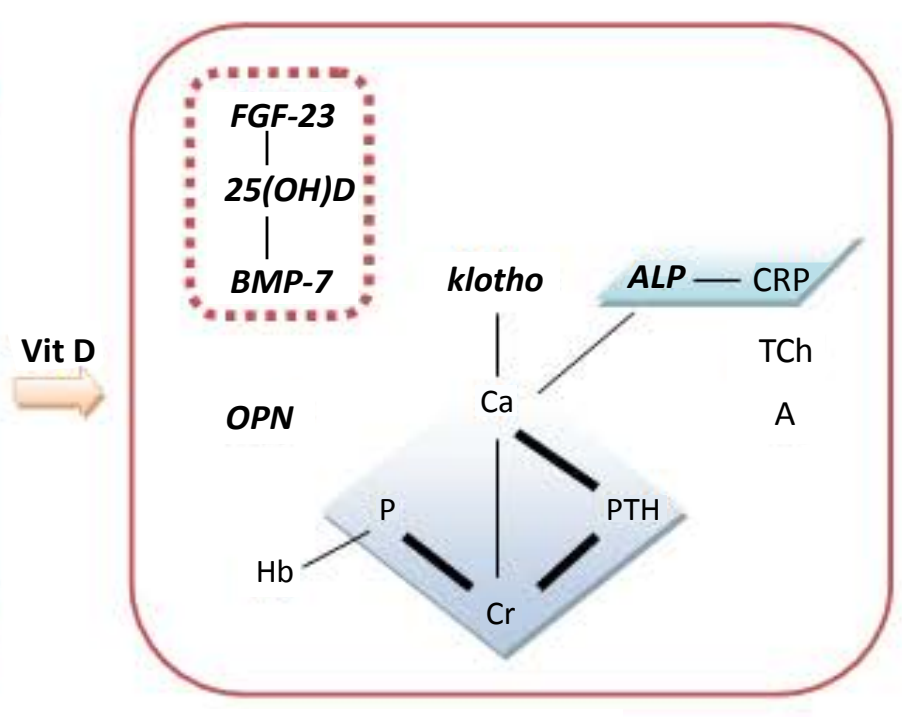

B

Figure 3: The correlations among blood biochemical parameters.

The relationships among parameters were tested by Linear Regression in month $0(A)$ and month 6 (B) during follow-up. The connecting lines represent the correlations among parameters. Compared to the baseline, the blood levels of $\mathrm{Ca}, \mathrm{P}, \mathrm{PTH}, \mathrm{SCr}$ and $\mathrm{Hb}$ had the similar correlations after 24 months of vitamin $\mathrm{D}$ treatment. The correlations among other parameters were not constant and changed after vitamin $D$ treatment.

Abbreviations: Hb: Hemoglobin; CRP: High Sensitive C Reactive Protein; A: Albumin; SCr: Serum Creatinine; TCh: Total Cholesterol; Ca: Calcium; P: Phosphorus; ALP: Alkaline Phosphatase; PTH: Intact Parathyroid Hormone; 25(OH)D: 25-hydroxyvitamin D; FGF: Fibroblast Growth Factor; BMP: Bone Morphogenetic Protein; OPN: Osteopontin; Vit D: Vitamin D treatment.

might be that ergocalciferol were metabolized in liver and changed to 25 hydroxyvitamin $\mathrm{D} 2$, while calcitriol has 1 and 25 hydroxl groups. The other reason might be that calcitriol prescription would be stopped temporarily when serum levels of $\mathrm{Ca}$ and/or $\mathrm{P}$ were beyond the target range according to the comments of guidelines $[3,4]$. Many studies show that calcitriol are more likely to cause hypercalcemia and hyperphosphatemia, which makes calcitriol have a narrow therapeutic window [15]. Comparably, ergocalciferol has fewer side effects than calcitriol in controlling $\mathrm{Ca}$ and $\mathrm{P}$ levels and can be used continuously in most CKD patients [13].

As well known, the bone metabolism biomarkers would change with kidney function in CKD patients. Accompanied with the elevation of SCr, the concentrations of iPTH and FGF-23 increased while klotho pro- tein and OPN levels decreased continuously and significantly during follow-up (Figure 2). However, the blood levels of iPTH, FGF-23, klotho protein and OPN have no significant differences between two treatment groups (Table 1 and Figure 2). These results demonstrated that bone metabolism hormones would change with renal dysfunction preceding other than with different vitamin $\mathrm{D}$ agents. The changing trends on these biomarkers were similar to the results of previous studies except for OPN $[8,16,17]$.

FGF-23-klotho system is an important regulator of calcium and phosphorus metabolism and plays a core role in development of CKD-MBD. FGF-23 is an endocrine hormone that is secreted by osteocytes, osteoblasts and other tissues than bone [18]. The primary physiological functions of FGF-23 are to stimulate phosphaturia 
Citation: Zhang D, Sun X, Li H (2018) Hormones of Bone Metabolism Changed with Vitamin D Agents Treatment in Chronic Kidney Disease Patients. J Hum Nutr 2(1):47-57

by down-regulating luminal expression of sodium-phosphate co-transporters in the proximal tubule, to reduce systemic levels of 1,25-dihydroxyvitamin $\mathrm{D}$ by directly inhibiting the renal 1-a hydroxylase and stimulating the catabolic 24-hydroxylase, as well as to inhibit PTH secretion. The classical effects of FGF-23 in the kidney and parathyroid glands are mediated by its binding to FGF receptors (FGFR) with the co-receptor klotho, which increases the binding affinity of FGF-23 for FGFR $[19,20]$. In the human kidney, decreased klotho expression occurs as early as in CKD stage 2. This decrease could be responsible, at least in part, for the early increase in serum FGF23 [5]. There was significant elevation on FGF-23 level and reducing on klotho protein level for 30 months follow-up (Table 1 and Figure 2), but neither SCr nor eGFR had correlation with FGF-23 or klotho protein in the present study (Table 2 and Figure 3). When we treat the CKD-MBD, calcitriol can promote the synthesis and secretion of klotho protein and the uptake of phosphorus [9]. However experimental studies have shown that calcitriol can also induce FGF-23 releasing from bones, particularly from the osteoblast lineage cells to elevate serum FGF-23 level [21,22]. Therefore, this replacement therapy of calcitriol is still in controversy. We compared the effects of calcitriol and ergocalferol on FGF-23 and soluble klotho levels in this study. The results identified that serum klotho protein decreased but FGF-23 increased significantly over time. There was no significant difference between two groups on either klotho protein or FGF-23 (Table 1 and Figure 2). So, we surmise that FGF-23 and klotho would be affected by many factors except for $\mathrm{SCr}$, especially in CKD 3-5 stage patients who were treated by vitamin D supplements. Moreover, 25( $\mathrm{OH}) \mathrm{D}$ level had a significantly negative correlation with FGF-23 after 24 months vitamin $\mathrm{D}$ treatment (Table 2), which suggested that vitamin D supplements could suppress endocrine of FGF-23.

OPN is a phosphoprotein expressed in the mineral tissues which inhibits mineralization by blocking hydroxyapatite formation and activating osteoclast function [23]. But the possibility that OPN could serve as a calcification serum marker is controversial. Barreto, et al. [16] demonstrated elevated plasma OPN levels in patients with CKD, even at early stages, in comparison to healthy volunteers. But one recent study did not find the differences of bone metabolism proteins, including of $\mathrm{OPN}$, fetuin A, osteocalcin, osteoprotegerin and matrix $\gamma$-carboxyglutamic acid protein, between CKD patients and control patients without CKD [17]. In the present study, we got different results. The OPN levels decreased significantly through following up period with the decrease of eGFR, and there were no significant differences between two groups (Figure 2P). These results may be caused by vitamin $\mathrm{D}$ treatment. However, the $25(\mathrm{OH}) \mathrm{D}$ was not an independent correlation factor of OPN in our study (Table 2). Then, more deep studies would be needed to do on OPN in CKD patients.

BMP-7 is an important factor in the formation of kidneys, eyes and bones. It plays an important role in bone metabolism. BMP-7 deficiency appeared in early stage of $\mathrm{CKD}$ and plays an important role in the pathogenesis of CKD and vascular calcification. Davies, et al. [24] found that BMP-7 can maintain the normal differentiation of VSMCs, up-regulate $\alpha$-smooth muscle actin expression, decline phosphorus levels, improve calcium cycling and calcification of the aorta in a rat model of atherosclerosis combined with CKD. Studies have demonstrated that BMP-7 can influence the number and morphology of osteoblasts, reduce the fibrosis surrounding trabecular, reduce bone resorption, and increase bone formation rate in SHPT. BMP-7 expression decreases after kidney damage. However, we did not find the decline trend in BMP-7 levels for 30 months follow-up. Otherwise, the circulating level of BMP-7 kept stable in Group aVitD3, and increased significantly in Group VitD2 (Table 1 and Figure 2O). Calcitriol is a stimulus of osteolysis, which may further promote BMP-7 expression to antagonize its effect. The present study suggested both ergocalciferol and calcitriol could maintain or even raise BMP-7 level in CKD patients. Linear regression results show BMP-7 level was correlated with $25(\mathrm{OH}) \mathrm{D}$ positively and significantly after vitamin $\mathrm{D}$ agents' treatment (Table 2 and Figure 3B). These indicated that improving $25(\mathrm{OH}) \mathrm{D}$ level would increase circulating BMP-7 level. And the effect of Vitamin D promoting bone formation by increasing BMP-7 may depend on raising blood levels of $25(\mathrm{OH}) \mathrm{D}$. The potential benefits of ergocalciferol on CKD-MBD by increasing BMP-7 are valuable to further research.

In this study, we also found SCr, P, iPTH and Ca had strong relationships between each other (Figure 3). Their correlations did not change through time, groups, $\mathrm{SCr}$ or eGFR levels, as well as vitamin D treatments. These results suggest blood $\mathrm{P}$ and $\mathrm{iPTH}$ concentrations were mainly determined by $\mathrm{SCr}$, and the change of $\mathrm{Ca}$ was crucially affected by iPTH.

For outcomes, clinical studies have demonstrated that excess FGF-23 led to an increase cardiovascular morbidity and mortality independently of the GFR in CKD patients [25-27]. Systemic klotho expression reduced with CKD progressing, circulating levels of klotho is connected with prognosis of CKD (creatinine level doubled, starting renal replacement therapy and death) [28]. However, Circulation level of klotho protein is not be related to kidney and does not predict adverse outcome in CKD patients in the study by Seiler, et al. [29]. For BMP-7, a nested case-control study of 281 patients 
Citation: Zhang D, Sun X, Li H (2018) Hormones of Bone Metabolism Changed with Vitamin D Agents Treatment in Chronic Kidney Disease Patients. J Hum Nutr 2(1):47-57

in the ADVANCE (Action in Diabetes and Vascular Disease: Preterax and Diamicron MR controlled Evaluation) study demonstrated the association between low levels of BMP-7 and poor renal outcomes (doubling of $\mathrm{SCr}, \mathrm{RRT}$, or death from renal disease) [30]. The positive association between plasma OPN level and clinical outcomes of CKD patients depended on their inflammatory status. Lau, et al. [9] demonstrated the up-regulated expression of OPN, a potent local inhibitor of vascular calcification, following administration of active vitamin $\mathrm{D}$. In the present study, there were no correlations between primary end-point events (ACD, AMI, stroke, CHF, and NTF) and bone metabolism biochemical parameters. For the secondary end-point events (the combining of the beginning of RRT, eGFR decline $>25 \%$, and doubling in SCr levels), $\mathrm{P}$ and iPTH levels were predicting factors.

There were several limitations in our study. In current knowledge, the ideal marker of CKD-MBD is limited. The pathophysiological mechanisms underlying this phenomenon are still poorly understood. This present study was designed to select several biomarkers which may play roles in CKD-MBD. We did not test the levels of BALP, which may be more special as a bone metabolism biomarker. We did not detect vascular calcification, which is a potent hazard during the use of vitamin D supplements. Though the vitamin D supplement effects of the ergocalciferol capsules could be differed from food sources of vitamin $\mathrm{D}$, we did not perform a diet survey and analysis.

In summary, the blood levels of bone metabolism hormones change significantly after vitamin D agents' therapy. Ergocalciferol supplementation can increase blood levels of BMP-7 with higher level of $25(\mathrm{OH}) \mathrm{D}$ compared to calcitriol. Improving $25(\mathrm{OH}) \mathrm{D}$ insufficiency can suppress the endocrine of FGF-23.

\section{Author Contributions}

Dongliang Zhang worked as the corresponding author to write this manuscript, design the research, and perform the research. Xiaodan Sun finished the following-up of patients in Beijing Rehabilitation Hospital of Capital Medical University, China. He Li collected the clinical and laboratory data.

Compliance with ethical standards.

\section{Funding}

There is no funding in this study.

\section{Conflict of Interest}

Author Dongliang Zhang declares that he has no conflict of interest. Author Xiaodan Sun, declares that she has no conflict of interest. Author He Li declares that she has no conflict of interest.

\section{Ethical Approval}

All procedures performed in studies involving human participants were in accordance with the ethical standards of the institutional and/or national research committee and with the 1964 Helsinki declaration and its later amendments or comparable ethical standards. Informed consent was obtained from all individual participants included in the study. The study protocol adhered to the Declaration of Helsinki and was approved by the institutional review boards of Beijing Friendship Hospital of Capital Medical University (NO. 2012-078).

\section{Registration of Clinical Trials}

This study is registered in ClinicalTrials.gov under accession no. NCT01633853.

\section{References}

1. Manraj Johal, Adeera Levin (2009) Vitamin D and Parathyroid Hormone in General Populations: Understandings in 2009 and Applications to Chronic Kidney Disease. Clin J Am Soc Nephrol 4: 1508-1514.

2. Zhang DL, Wang LY, Sun F, et al. (2014) Is the dialysis calcium concentration of $1.75 \mathrm{mmlo} / \mathrm{L}$ suitable for Chinese patients on maintenance hemodialysis? Calcif Tissue Int 94: 301-310.

3. National Kidney Foundation (2003) K/DOQI clinical practice guidelines for bone metabolism and disease in chronic kidney disease. Am J Kidney Dis 42: S1-S201.

4. Kidney Disease: Improving Global Outcomes (KDIGO) CKDMBD Work Group (2009) KDIGO clinical practice guideline for the diagnosis, evaluation, prevention, and treatment of Chronic Kidney Disease-Mineral and Bone Disorder (CKD-MBD). Kidney Int 113: S1-S130.

5. John GB, Cheng CY, Kuro-o M (2011) Role of Klotho in aging, phosphate metabolism, and CKD. Am J Kidney Dis 58: $127-134$

6. El-Abbadi MM, Pai AS, Leaf EM, et al. (2009) Phosphate feeding induces arterial medial calcification in uremic mice: role of serum phosphorus, fibroblast growth factor-23, and osteopontin. Kidney Int 75: 1297-1307.

7. Johan Lorenzen, Robert Krämer, S.M. Bode-Boeqer, et al. (2010) Circulating levels of osteopontin are closely related to glomerular filtration rate and cardiovascular risk markers in patients with chronic kidney disease. Eur J Clin Invest 40: 294-300.

8. Karakan S, Sezer S, Özdemir Acar FN, et al. (2011) Circulating levels of osteopontin are related with calcification parameters in patients with renal transplantations. Transplant Proc 43: 562-564.

9. Lau WL, Leaf EM, Hu MC, et al. (2012) Vitamin D receptor agonists increase klotho and osteopontin while decreasing aortic calcification in mice with chronic kidney disease fed a high phosphate diet. Kidney Int 82: 1261-1270.

10. Kaneko I, Saini RK, Griffin KP, et al. (2015) FGF-23 gene regulation by 1,25 -dihydroxyvitmin $D$ : opposing effects in adipocytes and osteocytes. J Endocrinol 226: 155-166. 
Citation: Zhang D, Sun X, Li H (2018) Hormones of Bone Metabolism Changed with Vitamin D Agents Treatment in Chronic Kidney Disease Patients. J Hum Nutr 2(1):47-57

11. Mehrotra R, Kermah D, Budoff M, et al. (2008) Hypovitaminosis $D$ in chronic kidney disease. Clin J Am Soc Nephrol 3: 1144-1151.

12. LaClair RE, Hellman RN, Karp SL, et al. (2005) Prevalence of calcidiol deficiency in CKD: A crosssectional study across latitudes in the United States. Am J Kidney Dis 45: 1026-1033.

13. Dongliang Zhang, He Li, Daoxin Yin, et al. (2016) Ergocalciferol versus calcitriolfor controlling chronic kidney disease mineral-bone disorder in stage 3 to 5 CKD: A randomized controlled trial. Eur J Pharmacol 789: 127-133.

14. Maunsell Z, Wright DJ, Rainbow SJ (2005) Routine isotope-dilution liquid chromatography-tandem mass spectrometry assay for simultaneous measurement of the 25-hydroxy metabolites of vitamins D2 and D3. Clin Chem 51: 1683-1690.

15. Shroff R, Egerton M, BridelM, et al. (2008) A bimodal association of vitamin $D$ levels and vascular disease in children on dialysis. J Am Soc Nephrol 19: 1239-1246.

16. Barreto DV, Lenglet A, Liabeuf S, et al. (2011) Prognostic implication of plasma osteopontin levels in patients with chronic kidney disease. Nephron Clin Pract 117: c363-372.

17. Anna Glub-Brzózka, Marta Michalska-kasiczak, Beata Franczyk, et al. (2016) Markers of increased atherosclerotic risk in patiens with chronic kidney disease: a preliminary study. Lipids in Health and Disease 15: 22.

18. Clinkenbeard EL, Cass TA, Ni P, et al. (2016) Conditional deletion of murine Fgf23: interruption of the normal skeletal responses to phosphate challenge and rescue of genetic hypophosphatemia. J Bone Miner Res 31: 1247-1257.

19. Goetz R, Ohnishi M, Kir S, et al. (2012) Conversion of a paracrine fibroblast growth factor into an endocrine fibroblast growth factor. J Biol Chem 287: 29134-29146.

20. Murali SK, Roschger P, Zeitz U, et al. (2016) FGF23 regulates bone mineralization in a $1,25(\mathrm{OH}) \mathrm{D}$ and klotho-independent manner. J Bone Min Res 31: 129-142.

21. Saini R, Kaneko I, Jurutka P, et al. (2013) 1,25-Dihydroxyvitamin D-3 regulation of fibroblast growth factor-23 expres- sion in bone cells: evidence for primary and secondary mechanisms modulated by leptin and interleukin-6. Calcif Tissue Int 92: 339-353.

22. Meir T, Durlacher K, Pan Z, et al. (2014) Parathyroid hormone activates the orphan nuclear receptor Nurr1 to induce FGF23 transcription. Kidney Int 86: 1106-1115.

23. Scatena M, Liaw L, Giachelli CM (2007) Osteopontin: a multifunctional molecule regulating chronic inflammation and vascular disease. Arterioscler Thromb Vasc Biol 27: 2302-2309.

24. Davies MR, Lund RJ, Mathew S, et al. (2005) Low turnover osteodystrophy and vascular calcification are amenable to skeletal anabolism in an animal model of chronic kidney disease and the metabolic syndrome. J Am Soc Nephrol 16: $917-928$

25. Seiler S, Reichart B, Roth D, et al. (2010) FGF-23 and future cardiovascular events in patients with chronic kidney disease before initiation of dialysis treatment. Nephrol Dial Transplant 25: 3983-3989.

26. Scialla J, Xie H, Rahman M, et al. (2014) Fibroblast growth factor-23 and cardiovascular events in CKD. J Am Soc Nephrol 25: 349-360.

27. Ämlöv J, Carlsson AC, Sundström J, et al. (2013) Serum FGF23 and risk of cardiovascular events in relation to mineral metabolism and cardiovascular pathology. Clin J Am Soc Nephrol 8: 781-786.

28. Hyoung Rae Kim, Bo Young Nam, Dong Wook Kim, et al. (2013) Cireculating $\alpha$-Klotho Levels in CKD and Relationship to Progression. Am J Kidney Dis 61: 899-909.

29. Seiler S, Wen M, Roth HJ, et al. (2013) Plasma Klotho is not related to kidney function and does not predict adverse outcome in patients with chronic kidney disease. Kidney Int 83: $121-128$.

30. Wong MG, Perkovic V, Woodward M, et al. (2012) Circulating bone morphogenetic protein-7 and transforming growth factor- $\beta 1$ are better predictors of renal end points in patients with type 2 diabetes mellitus. Kidney Int 83: 278-284. 